\title{
Timosaponin-Bll inhibits the up-regulation of BACE1 induced by Ferric Chloride in rat retina
}

\author{
Ju-Fang Huang ${ }^{1}$, Lei Shang ${ }^{1}$, Pei Liu², Meng-Qi Zhang ${ }^{3}$, Shuang Chen', Dan Chen', Chun-Ling Fan', \\ Hui Wang ${ }^{1}$ and Kun Xiong ${ }^{1 *}$
}

\begin{abstract}
Background: Our previous studies indicated that oxidative stress up-regulated the expression of $\beta$-amyloid precursor protein cleavage enzyme-1 (BACE1) in rat retina. Pharmacological reports have shown Timosaponin-Bll, a purified extract originating from Chinese medical herb Rhizoma Anemarrhenae, is characterized as an antioxidant. Our present study aimed to determine whether Timosaponin-Bll affected the expression of BACE1, $\beta$-amyloid precursor protein cleavage production of $A \beta 1-40$ and $\beta-C$-terminal fragment ( $\beta-C T F)$ in rat retina, which were pre-treated with the oxidizing agent (solution of $\mathrm{FeCl}_{3}$ ).

Results: Few distinctions of BACE1 distribution were observed among all groups (normal control group, model group, Timosaponin-Bll treated and vehicle control groups). Rat retinas in model group and vehicle control group manifested an apparent up-regulation of BACE1 expression. Meanwhile, the level of malonaldehyde (MDA), A $1-40$ and $\beta-C T F$ were increased. However, when comparing with the vehicle control group, the retinas in Timosaponin-Bll treated group showed significantly less BACE1 $(p<0.05)$ and accumulated less A $1-40$ or $\beta-C T F$ $(p<0.05)$. It also showed significantly decreased level of MDA $(p<0.05)$ and prolonged partial thromboplastin time $(p<0.05)$.
\end{abstract}

Conclusion: Our data suggested that Timosaponin-BII remarkably inhibited the up-regulation of BACE1 and reduced the over-production of $\beta-C T F$ and $A \beta$ in rat retina, which was induced by $\mathrm{FeCl}_{3}$. The mechanism of Timosaponin-BII on BACE1 expression may be related to its antioxidant property.

Keywords: Timosaponin-BII, Oxidative stress, BACE1, Retina, AB

\section{Background}

Alzheimer's disease (AD) is a progressive neurodegenerative disease prevalent in aged populations and characterized by the presence of senile plaques (SP), neurofibrillary tangles (NTFs), capillary cerebral amyloid angiopathy (capCAA) and the abnormal loss of synapses $[1,2]$. The amyloid deposition, whose disposal mechanisms have attracted great attention to neurologists, is regarded as a main etiology. It has been documented that accumulation of amyloid $\beta$-peptide $(A \beta)$, which is a cleavage form of $\beta$-site amyloid precursor protein ( $\beta$ APP), acts as a fundamental initiator of this disease [3]. $\beta$-APP is cleaved by $\beta$-amyloid precursor protein cleavage enzyme-1 (BACE1), along with the digestion by $\gamma$ -

\footnotetext{
* Correspondence: xiongkun2001@163.com

'Department of Anatomy and Neurobiology, School of Basic Medical Sciences, Central South University, Changsha, Hunan 410013, China Full list of author information is available at the end of the article
}

secretase, which results in the release of $A \beta$ [4]. Therefore, BACE1 plays a significant role in secretion of $A \beta$. Previous studies have shown that different pathological conditions, including traumatic brain injury, cerebral ischemia, oxidative stress, functional deprivation, lead exposure, ischemia and hypoxia, contribute to the upregulation of BACE1 expression or facilitate its activity, and eventually lead to the massive accumulation of $A \beta$ [5-11]. Our previous data showed that oxidative stress induced by the intra-vitreous injection of $\mathrm{FeCl}_{3}$ solution elevated the expression of BACE1 in rats' retina, and consequently triggered the dramatic production of $A \beta$ [12]. This finding is in agreement with other studies [1315]. It is generally accepted that up-regulation of BACE1 expression as well as the degree of activated BACE1 is closely related to the aggravation and the onset of $\mathrm{AD}$ $[16,17]$. Therefore, investigations focused on inhibiting either excessive generation or hyperactivity of BACE1 
are extensively established on account of alleviating the symptoms or even eradicating of the disease in early stage.

Zhimu, a traditional Chinese medicine, is derived from the dry rhizome of the plant Anemarrhena asphodeloides $B g e$, Numerous steroids have been extracted from this plant, such as sarsasapogenin, markosapogenin, negitogenin, and their glycosylated products named TimosaponinAI, -AII, -AIII, -AIV, -BI and -BII [18]. It has been reported that Timosaponin-BII has a neuronal protective and anti-inflammatory effect possibly by suppressing the production of pro-inflammatory factors IL-1, IL-6 and TNF- $\alpha$ [19-21]. The dementia-palliative effect of Timosaponin-BII may involve multiple mechanisms and one of them is its potential anti-oxidative property. An intriguing observation from Ouyang's experiment showed that Timosaponin-BII diminished the $A \beta$ induced oxidative impairment by promoting scavenging of superoxide radicals [22]. Nevertheless, it is to be elucidated whether this neuronal protective effect would inhibit the oxidant-induced up-regulation of BACE1 expression and even would attenuate the overproduction of $\mathrm{A} \beta$ with its anti-oxidant property. In the present study, we constructed an oxidative impairment animal model by intra-vitreous injection of $\mathrm{FeCl}_{3}$ solutions then administered Timosaponin-BII to these animal models. The current study aimed to determine whether Timosaponin-BII could inhibit the production of BACE1 in the retina including $A \beta$ and malondialdehyde (MDA), which is a lipid oxidative product.

\section{Methods}

\section{Reagents and Animals}

Reagents

The powder of Timosaponin-BII was provided by Professor Wan-Sheng Chen from Department of Pharmacology, School of Pharmacology, Second Military Medical University, Shanghai, China [23] and its purity is above $98 \%$. Timosaponin-BII was dissolved in normal saline (NS) at $1.2 \mathrm{~g} / \mathrm{ml}$ in room temperature $\left(24^{\circ} \mathrm{C}\right)$, subsequently diluted $\mathrm{FeCl}_{3}$ (Sigma, MO, USA) with $0.01 \mathrm{M}$ PBS at $10 \mathrm{mM}$ to yield a low concentration working solutions. The working concentration to induce the proper increase of BACE1 expression and A $\beta$ aggregation has been determined in our previous study [12].

\section{Animals}

Twenty-four adult male SD rats weighing 200-250 g and aged 2-3 months were purchased from Animal Center of Xiangya School of Medicine, Central South University. All rats were isocoria with eyes observed transparent and no deformity. The laboratory animals were housed in a constant temperature at $24^{\circ} \mathrm{C}$ with relative humidity at $(55 \pm 10) \%$. All experiments were carried out according to the principles outlined in the NIH Guide for Care and Use of Laboratory Animals. The present study was approved by Animal Ethics Committee of Xiangya School of Medicine, Central South University.

\section{Animal model construction and drug administration}

Rats were equally divided into four groups randomly: The normal control group (CTL), the model group $\left(\mathrm{FeCl}_{3}\right)$, the vehicle control group $\left(\mathrm{FeCl}_{3}+\mathrm{NS}\right)$ and the experimental group $\left(\mathrm{FeCl}_{3}+\right.$ Timosaponin-BII). The methods of constructing models were previously described in detail in our paper [12], and briefly described as follows: We anaesthetized the rats with chloral hydrate by peritoneal injection and fixed them at stereotaxic apparatus with eyes properly exposed. Afterwards, we extracted equal amount of humor vitreous and injected the $\mathrm{FeCl}_{3}$ solution at $5 \mu \mathrm{l}$ with a needle penetrating the eyeball from the dorsal sclera at $1 \mathrm{~mm}$ posterior to the cornea-sclera junction and toward the center of the eye for $2 \mathrm{~mm}$ deep. The procedure was cautiously carried out to avoid penetrating the beyond lens. The experimental group received Timosaponin-BII at $6 \mathrm{mg} / \mathrm{kg}$ by intravenous injection every morning for 14 days [24] while the vehicle control group was given an injection of equal amount of normal saline. No drugs were administered to the normal control or model group. At the $14^{\text {th }}$ day, we randomly selected three in each group for morphological studies while the remaining animals were used for biochemistry studies.

\section{Prothrombin time assay}

Blood samplings were collected by puncturing the heart using natrium citricum negative pressure tubes that was provided by Department of Laboratory, the Third Xiangya Hospital of Central South University. The rats were deeply anaesthetized with chloral hydrate and their chests were promptly opened in order to expose the heart. Consequently we punctured the left artrium with a negative pressure tube. Three milliliters of blood samples were collected from the experimental group and the vehicle control group for the determination of Prothrombin time (PT), Activated partial thromboplastin time (APTT), Thrombin time (TT) and the concentration of Fibrinogen (FIB). Subsequently, the rats were transcardiacally perfused.

\section{Immunofluorescence staining}

The eyeballs were removed following trans-cardiac perfusion with $4 \%$ paraformaldehyde in PBS at certain survival time point, post-fixed in the perfusion solution overnight, followed by cryo-protection in $30 \%$ sucrose. The eyeballs were cut in a cryostat at $14 \mu \mathrm{m}$ thickness. Cross-retinal sections near and passing the optic nerve 
head were thaw-mounted on Superfrost Plus slides (VWR, PA, USA).

Sections were incubated overnight at $4{ }^{\circ} \mathrm{C}$ either with BACE1 antibody (1:200; polyclone, AB5940, Millipore, MA, USA) after blocked by PBS solution containing $10 \%$ BSA and $0.3 \%$ Triton X-100 (Sigma, MO, USA) for $1 \mathrm{hr}$. Immunoreaction products were visualized following $2 \mathrm{hr}$ incubation with Alexa Fluor ${ }^{\circledR} 568$ conjugated donkey anti-rabbit IgG (1:200; Invitrogen, CA, USA). Sections were counterstained with Hoechst 33342 (1:50k; Sigma, MO, USA), washed, and mounted with anti-fading medium before microscopic examination. Digital images were obtained using fluorescence microscopy (Olympus, BH-40, Tokyo, Japan).

\section{Western blotting}

For biochemical studies, the eyeballs were removed following a vascular rinse with ice cold PBS under overdose anesthesia (sodium pentobarbital $100 \mathrm{mg} / \mathrm{kg}$, i.p.) in each group. The retinas were dissected out in cold PBS on ice, carefully soaked with a piece of filter paper, then collected in $1.5 \mathrm{ml}$ test tubes and weighed. The retinas were homogenized by sonication in cell lysis buffer $(10 \times$ w/v, Pierce Inc., CA, USA) containing a cocktail of protease inhibitors (Roche, IN, USA). Tissue lysates were centrifuged (Ependoff, CA, USA) at $15,000 \times g$ at $4^{\circ} \mathrm{C}$ for $15 \mathrm{~min}$ after standing on ice for $1 \mathrm{hr}$. Supernatants were collected and protein concentrations were determined by BCA protein assay (Pierce Inc., CA, USA). Equal quantity of protein $(40-100 \mu \mathrm{g}$ depending on target proteins) was run on $10 \%$ SDS-PAGE gel (Bio-Rad, CA, USA) after protein denaturation. The polypeptides were electro-transferred to Trans-Blot $^{\circledR}$ pure nitrocellulose membrane (Bio-Rad, CA, USA). Non-specific binding was blocked with PBS containing 5\% non-fat milk. Nitrocellulose membranes were incubated with primary antibodies to BACE1, APP (1:500; polyclone, AHP538, Serotec, CA, USA) and $\beta$-tubulin (1:10k; polyclone, Sigma, MO, USA) overnight in $4^{\circ} \mathrm{C}$. Membranes were further incubated in HRP-conjugated secondary antibodies (1:20k; Bio-Rad, CA, USA) for $1 \mathrm{hr}$. Protein bands were visualized with an ECL Plus ${ }^{\mathrm{TM}}$ Western Blotting Detection kit according to manufacturer's instruction (GE Healthcare Life Sci., NJ, USA).

\section{A 1-40 ELISA Assay}

A $\beta$ 1-40 levels in retina extracts were assayed using a commercial kit according to manufacturer's instruction (Biosource International, CA, USA), except that the reporter antibody supplied by the manufacturer was replaced with biotinylated 4G8 at 1:4k (Signet Laboratories Inc., MA, USA). Equal quantity $(80 \mu \mathrm{g})$ of protein was loaded in each well and each analysis performed in duplicate.

\section{MDA concentration assay}

MDA levels in retina extracts were assayed using a commercial kit according to manufacturer's instruction (Jian-Cheng Biotechnical Co., Nanjing, China), the standard reference substance named tetraethoxypropane were used in $10 \mathrm{nmol} / \mathrm{ml}$. Equal quantity $(100 \mu \mathrm{g})$ of protein was loaded in each well and each analysis performed in duplicate.

\section{Statistic analysis}

Specific density was calculated by subtracting background density on the membrane from total density measured over the protein band, followed by standardization to $\beta$-tubulin references using the software of Image J (Image J, MD, USA). For ELISA data, levels of $A \beta 1-40$ were determined by a standard curve generated using serially diluted synthetic $A \beta 1-40$ peptide provided in the kit. Finally, all data were normalized to the means of inner reference (for western blotting) or the means of control group (for ELISA data) whenever appropriate, yielding relative values expressed as percentages of controls (control serve as 100\%).

Statistical analyze of normalized values were conducted using one-way ANOVA followed by PASW SPSS 19.0 (SPSS, CA, USA), yielding $p$ values between individual groups. The data expressed as means \pm SD. The minimal significant level of difference between groups was set at $p<0.05$.

Digital images of retinal immunolabeling were captured at $40 \times$ objectives at comparable retinal segments ( $\sim 0.5 \mathrm{~mm}$ apart from the optic head). All illustrations were prepared with Adobe Photoshop CS 6.0 (Adobe, CA, USA). Whole panel images were converted to TIFF format, and contrast/brightness was adjusted if necessary.

\section{Results}

\section{Timosaponin-Bll prolonged APTT}

When compared with the vehicle control, TimosaponinBII treated rats had no difference in TT, PT and the concentration of FIB while the APTT was significantly longer (Figure 1). This result indicated that Timosaponin-BII had an inhibitory effect on intrinsic coagulation system, which is in agreement with a previous study of Lu et al. (2011) [24].

\section{Timosaponin-Bll decreased BACE-1 expression in rat retina}

The immunofluorescence staining result showed that BACE1 was mainly present in inner plexiform layer (IPL), outer plexiform layer (OPL) and the nerve fiber layer (NFL). Slight cellular dispersion also existed in the inner and out nuclear layers. These results were consistent with our previous studies [12]. In contrast with the normal 


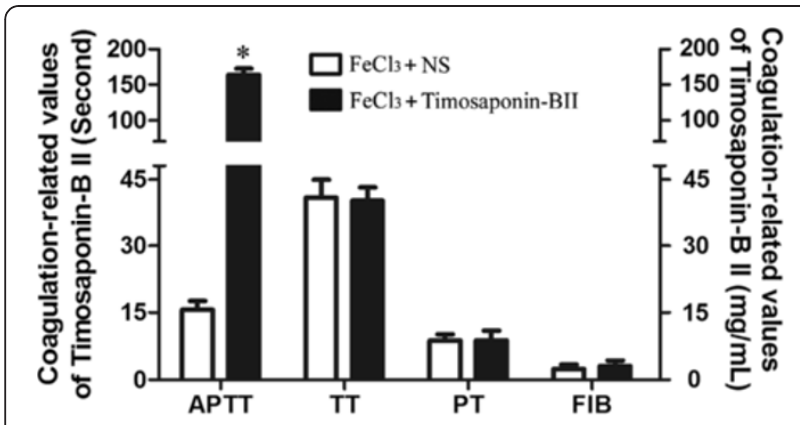

Figure 1 Effect of Timosaponin-BII on Prothrombin time (PT). Activated partial thromboplastin time (APTT), Thrombin time (TT) and the concentration of Fibrinogen (FIB). * : compared with vehicle control group: $p<0.05, \mathrm{n}=3$.

control, the $\mathrm{FeCl}_{3}$-treated animal model showed significantly more distinct and heavier BACE1 immunoreactivity and exhibited no apparent discrepancies in the cellular and laminar distribution of BACE1. The vehicle control showed a similar expression pattern as the model group. Although no difference in distribution was detected, the experimental group displayed remarkably lower immunoreactivity when compared with the model group and the vehicle control, but it remained higher than the normal control (Figure 2).

The western blotting results showed that BACE1 was mainly exhibited as a single $46 \mathrm{kDa}$ band in all groups. The bands in model group as well as in the vehicle control group were apparently thicker and larger than those of normal control group. The band in the experimental group was thinner and smaller than those in model group and vehicle control but remained larger than the normal ones (Figure 3A).

\section{Timosaponin-Bll decreased the overproduction of $\beta-C T F$ in rat retina}

$\beta$-CTF (BACE1 cleavage product) and $\beta$-tubulin were mainly exhibited as a single $14 \mathrm{kDa}$ band or $55 \mathrm{kDa}$ band respectively in all the four groups. The bands of $\beta$ CTF in model group as well as the vehicle control group

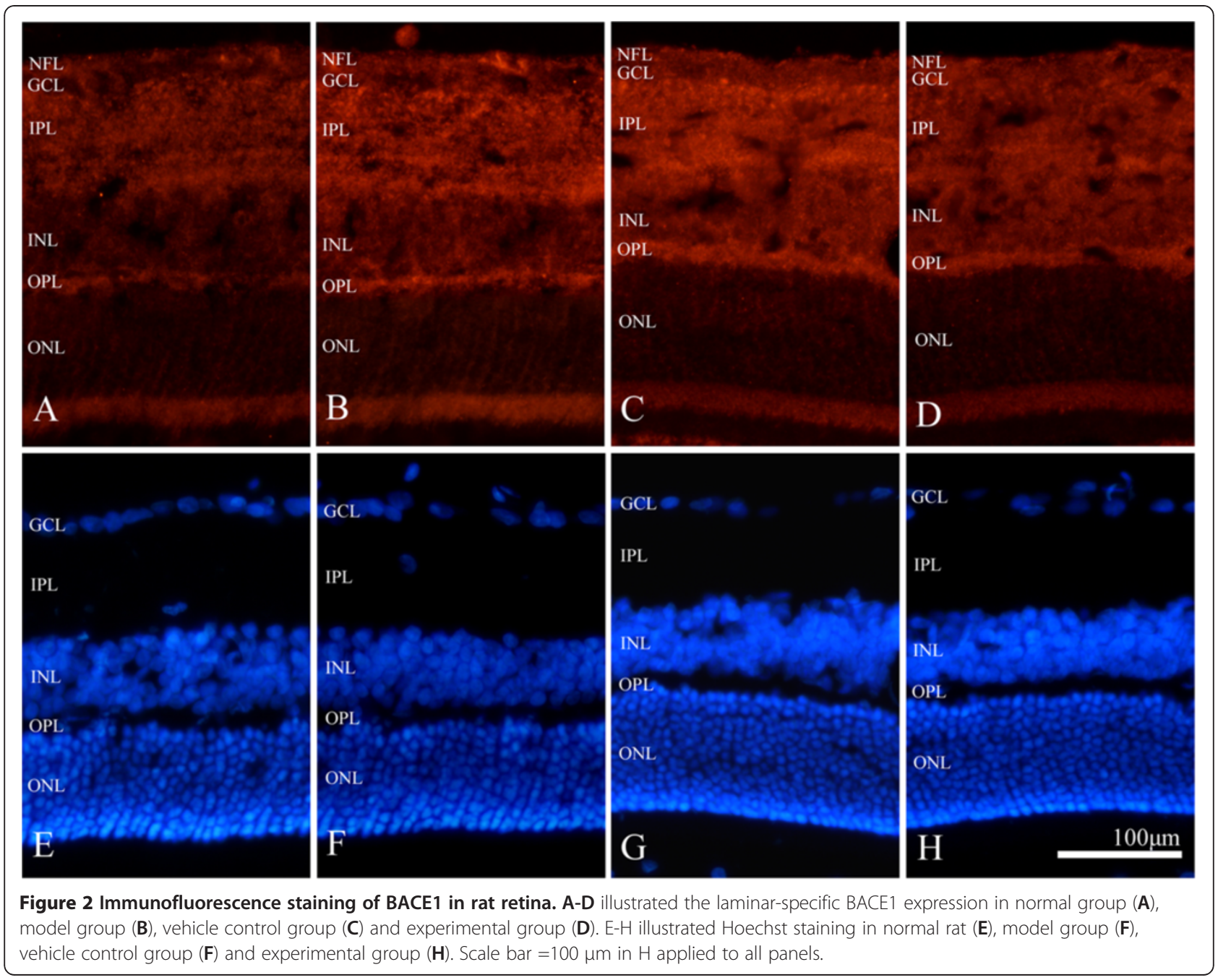




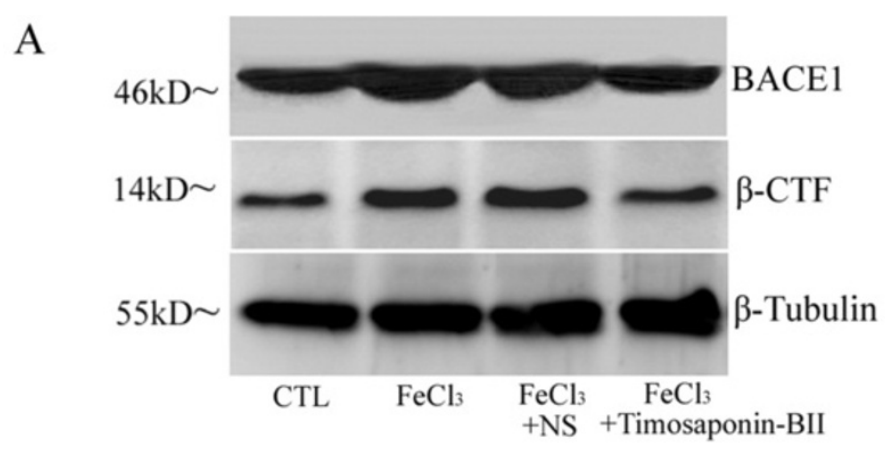

$\mathrm{B}$

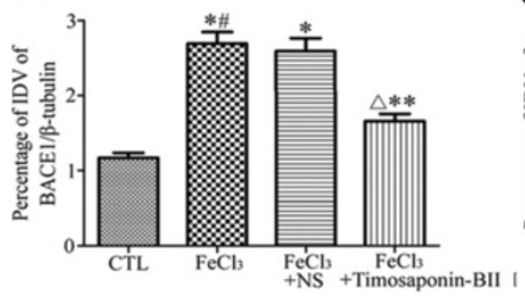

$\mathrm{C}$

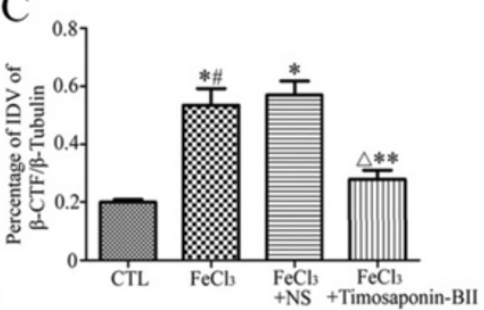

Figure 3 Western blotting analysis result of BACE1 and $\beta$-CTF. A: bands of BACE1, $\beta-C T F$ and $\beta$-tubulin; B: optical density analysis of BACE1; C: optical density analysis of $\beta$-CTF. *: compared with normal control group: $p<0.05$; \#: compared with vehicle control group: $p>0.05 ; \Delta$ : compared with normal control group: $p<0.05$; **: compared with model groups or vehicle control group: $p<0.05, \mathrm{n}=3$.

were apparently thicker and larger than normal control. The bands in the experimental group were thinner and smaller than those in model group and vehicle control but remained larger than the normal ones (Figure 3A).

The optical density measurement and statistical analysis indicated that the vitreous injection of $\mathrm{FeCl}_{3}$ upregulated the expression of BACE1 and resulted in the overproduction of $\beta$-CTF. The vehicle control group showed a similar result with the model group. The rats that were administered with Timosaponin-BII apparently manifested a significant decrease in BACE1 expression and $\beta$-CTF production in relation to the model group and the vehicle control groups but still remained higher than the normal ones (Figure 3B, C).

\section{Timosaponin-BII inhibited overproduction of $A \beta$ in rat retina}

The statistics analysis of ELISA result for $A \beta 1-40$ level was described in Figure 4. No discrepancies in A $\beta 1-40$ concentration were observed between the model group and the vehicle control group but both the two groups showed the upsurge in relation to the normal control group. The level of $A \beta 1-40$ in Timosaponin-BII treated group was lower than those in model group and vehicle group but remained higher than those in the normal control group. This result indicated that TimosaponinBII remarkably inhibited overproduction of $A \beta$ that was induced by up-regulation of BACE1. Nevertheless, the effect of Timosaponin-BII was limited in extent since the level of target peptides was still higher than those in normal condition.

\section{Timosaponin-Bll reduced MDA production in rat retina}

The statistics analysis of MDA level is shown in Figure 5. No discrepancies in MDA concentration were observed between the model group and the vehicle control group but both groups showed the upsurge in relation to the normal control group. The level of MDA in the experimental group was lower than those in model group and vehicle control group but remained higher than those in the normal control group. This result indicated that

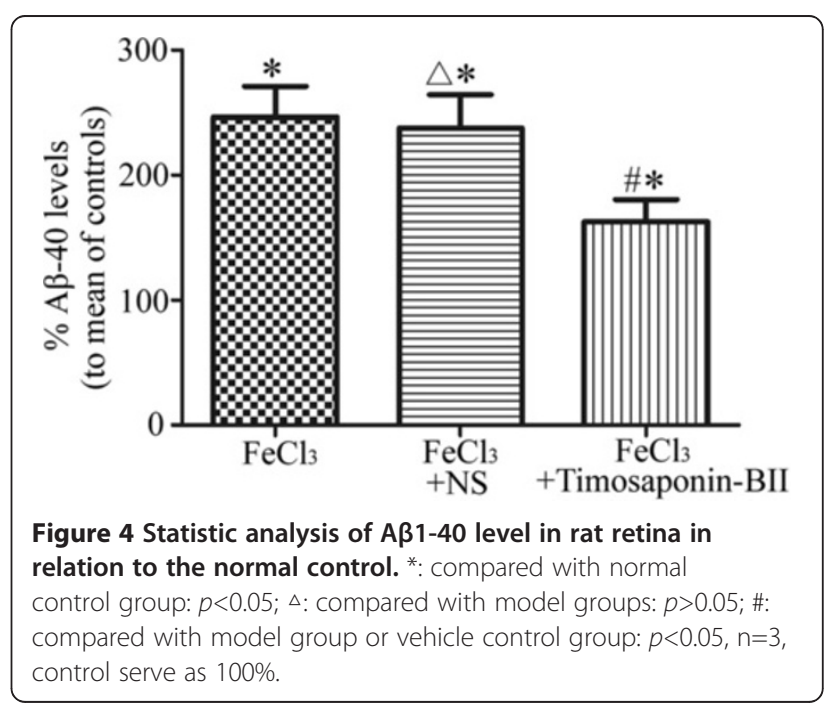




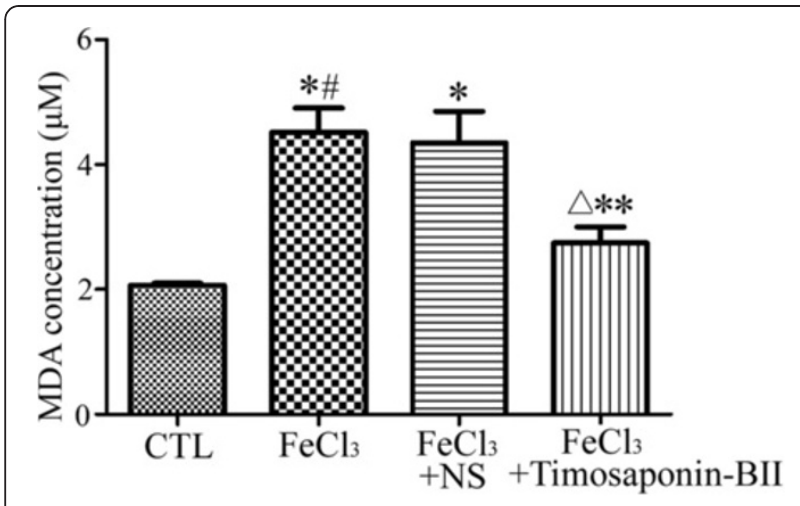

Figure 5 Statistical analysis of MDA level in rat retina. *: compared with normal control group: $p<0.05$; \#: compared with vehicle control group: $p>0.05 ; \Delta$ : compared with normal control group: $p<0.05$; ${ }^{* *}$ : compared with model group or experimental group: $p<0.05 . n=3$.

Timopaponin-BII reduced the oxidative stress that was induced by the $\mathrm{FeCl}_{3}$ in rats' retina. Nonetheless, the capacity was limited as the oxidative stress remained higher in the normal control group.

\section{Discussion}

Alzheimer's disease (AD) is a progressive neurodegenerative disease, which is characterized as deterioration of memory and global cognitive ability, in addition to the impairment of language skills and the extreme apathy and exhaustion $[25,26]$. Senile plaques, neurofibrillary tangles, capillary cerebral amyloid angiopathy and the abnormal loss of synapses are proposed to be the principal pathological changes in $\mathrm{AD}[1,2]$. Moreover, the amyloid plaque originating from the $\beta$-APP abnormal proteolysis product $A \beta$ is considered to be the predominant feature of the disease. In addition to the frontal lobe, occipital lobe and the hippocampus that were traditionally identified with pathological molecular abnormalities in $\mathrm{AD}$, retina has recently been documented to be equivalently involved in the morbidity process [27]. Some specialists point out that the detectable $A \beta$ would turn up in patients' retina in the early stages rather than advanced ones, which provides pre-diagnostic evidence before the occurrence of dementia [28]. The pertinent experiment carried out on animals also reaches a similar conclusion. Over-production of $A \beta$ and $\beta$-amyloid plaques in retina were detected prior to behavior transformation and cognitive impairment in $\mathrm{AD}$-transgenic animals $[28,29]$. Dependent on aforementioned facts and data, we selected retina as an experimental object to explore the effect of Timosaponin-BII on $A \beta$ laid in this conclusion and relevant evidence about $A \beta$ presence inferred from our previous studies [11,12].

Oxidative stress has long been unequivocally esteemed as one of the principal etiological factors of AD [30-33].
Related reports illustrated that $\mathrm{A} \beta$ had an oxidative toxicity for neurons [34], while current evidence supports the amplifying effect of oxidative stress on A $\beta$ aggregation [7]. Thus, a complete self-enforcing circle including A $\beta$ accumulation and reactive oxygen production eventually resulted in neuronal death. Our previous data revealed that the $\mathrm{FeCl}_{3}$ induced oxidative stress upregulated BACE1 expression [12], compatible with Tamagno's conclusion that oxidative stress promoted BACE1 activation in NT2 cells [15]. In our present study, the apparent increase of BACE1 protein and cleavage product $\mathrm{A} \beta$, as well as $\mathrm{MDA}$ in $\mathrm{FeCl}_{3}$ treated retina indicated the success in constructing the oxidative model.

Timosaponin-BII is widely accepted as neuronal protective chemicals extracted from Chinese traditional herb (Anemarrhena asphodeloides). Nevertheless, the mechanisms of its prevention for neurons from injury still remain to be further elucidated. Several primary hypotheses contribute to this issue. First of all, Timosaponin-BII may inhibit the inflammation in nerve system under pathological conditions in the context of its inhibitory effect on the generation of proinflammatory factors IL-1, IL-6 and TNF [20,21,35]. Second, Timosaponin-BII partly restores rats' intelligence quality that is suppressed by $A \beta$ aggregation through the ameliorating activity of acetylcholine esterase (AchE) [36]. Third, some specialists believe that anti-platelet and antithrombotic activities of Timosaponin-BII potentially prevent the recurrence of thrombus formation and preserve cerebral blood flow, contributing its neuroprotective effect against cerebral ischemia dementia [24,35]. Although little involvement of Timosaponin-BII in extrinsic coagulation system was observed, Lu's experiment evidently demonstrated its inhibition effect on intrinsic coagulation system. Timosaponin-BII may prevent against the formation and extension of blot clots, which consequently preclude cognitive impairment from ischemia. Our result was in agreement with Lu's conclusions, but whether the anti-platelet effect influence the blood supply of retina still remains to be investigated. Furthermore, Timosaponin-BII may reduce the direct damage to neurons with its anti-oxidative property [22]. The present data showed that the application of Timosaponin-BII to oxidant injury model led to the decrease of BACE1 protein and $A \beta$, together with the oxidative product (MDA) in vivo. This abatement of BACE1 production associated with the degraded level of MDA in Timosaponin-BII treated animals examined by our group and others. These similar observations may raise conceptual concern to the underlying neuroprotective potential of Timosaponin-BII in oxidative-stressrelated aggregation of $A \beta$ in $A D$ pathology. It is tempting to presume that Timosaponin-BII not only prevents the 
direct attack to neurons from reactive oxygen but also inhibits the process of the "oxidative stress-BACE1$A \beta$-neuronal damage" initially with its anti-oxidative capacity, finally resulting in a neuroprotective effect.

It is worthwhile to note with regard to the level of reactive oxygen, BACE1 protein and overproduction of $A \beta$ still remains higher than those of normal control. We believe that the mass of acute and intensive impairment in retina induced with immediate oxidant injection elevates the level of BACE1 protein and $A \beta$ in a short time, while the anti-oxidative capability of Timosaponin-BII prevents further injury caused by reactive oxygen species rather than eliminating the preexisting pathological molecule, leaving $A \beta$ aggregation in the tissue unvarnished. Additionally, the discrepancy could also be attributed to the complexity in the mechanisms of oxidative stress and the limited competence of Timosaponin-BII as a single monomer from nature plants. At present, there are some limitations of the effects of Timosaponin-BII. However, in terms of its predominant merit in reducing level of oxidant stress and inhibiting the up-regulation of BACE1, our results suggested Timosaponin-BII had the neuroprotective potential through interference with molecules related to $A \beta$ production.

\section{Conclusion}

This present study indicated that Timosaponin-BII inhibited the up-regulation of BACE1 caused by $\mathrm{FeCl}_{3}$ in rats retina and reduced the production of $\mathrm{A} \beta$ presumably with its anti-oxidant potentials.

\section{Competing interests}

The authors declare that they have no competing interests.

\section{Authors' contributions}

$\mathrm{KX}$ and J-FH designed the experiment. LS, PL and SC performed the experiment. PL and M-QZ drafted the manuscript. HW, C-LF and DC analyzed the data. $K X$ and $D C$ revised the manuscript and participated in paper modification. All authors participated in critical revision of the manuscript and approved the final manuscript.

\section{Acknowledgements}

This work is supported by National Natural Science Foundation of China (Grant \# 30900773; Grant \# 81070729), Natural Science Foundation of Hunan Province (Grant \# 11 JJ2020) and Young Teachers Training Program of Normal University of Hunan Province to X. K. We are grateful for the provision of Timosaponin-Bll from Professor Wan-Sheng Chen in Department of Pharmacology, School of Pharmacology, Second Military Medical University, Shanghai, China. Thanks for Dr. George Tipoe (Department of Anatomy and Ophthalmology, Li Ka Shing Faculty of Medicine, The University of Hong Kong) to help us copyedit the manuscript.

\section{Author details}

'Department of Anatomy and Neurobiology, School of Basic Medical Sciences, Central South University, Changsha, Hunan 410013, China. ${ }^{2}$ Five-year Medicine Program, Grade 2009, Central South University Xiangya School of Medicine, Changsha, Hunan 410013, China. ${ }^{3}$ Eight-year Clinical Medicine Doctor Program, Grade 2006, Central South University Xiangya School of Medicine, Changsha, Hunan 410013, China.
Received: 29 June 2012 Accepted: 18 October 2012

Published: 22 October 2012

\section{References}

1. Attems J, Yamaguchi H, Saido TC, Thal DR: Capillary CAA and perivascular Abeta-deposition: two distinct features of Alzheimer's disease pathology. J Neurol Sci 2010, 299(1-2):155-162.

2. Gotz J, Eckert A, Matamales M, Ittner LM, Liu X: Modes of Abeta toxicity in Alzheimer's disease. Cellular and molecular life sciences: CMLS 2011, 68(20):3359-3375.

3. Selkoe DJ: Toward a comprehensive theory for Alzheimer's disease. Hypothesis: Alzheimer's disease is caused by the cerebral accumulation and cytotoxicity of amyloid beta-protein. Ann N Y Acad Sci 2000, 924:17-25.

4. Selkoe DJ: Alzheimer's disease: genes, proteins, and therapy. Physiol Rev 2001, 81(2):741-766.

5. Blasko I, Beer R, Bigl M, Apelt J, Franz G, Rudzki D, Ransmayr G, Kampfl A, Schliebs R: Experimental traumatic brain injury in rats stimulates the expression, production and activity of Alzheimer's disease beta-secretase (BACE-1). J Neural Transm 2004, 111(4):523-536.

6. Wen $Y$, Onyewuchi $O$, Yang S, Liu R, Simpkins JW: Increased beta-secretase activity and expression in rats following transient cerebral ischemia. Brain Res 2004, 1009(1-2):1-8.

7. Tong Y, Zhou W, Fung V, Christensen MA, Qing H, Sun X, Song W: Oxidative stress potentiates BACE1 gene expression and Abeta generation. J Neural Transm 2005, 112(3):455-469.

8. Velliquette RA, O'Connor T, Vassar R: Energy inhibition elevates betasecretase levels and activity and is potentially amyloidogenic in APP transgenic mice: possible early events in Alzheimer's disease pathogenesis. J Neurosci 2005, 25(47):10874-10883.

9. Sun X, He G, Qing H, Zhou W, Dobie F, Cai F, Staufenbiel M, Huang LE, Song W: Hypoxia facilitates Alzheimer's disease pathogenesis by up-regulating BACE1 gene expression. Proc Natl Acad Sci U S A 2006, 103(49):18727-18732.

10. Yan XX, Xiong K, Luo XG, Struble RG, Clough RW: beta-Secretase expression in normal and functionally deprived rat olfactory bulbs: inverse correlation with oxidative metabolic activity. J Comp Neurol 2007, 501(1):52-69.

11. Huang JF, Huang $K$, Shang L, Wang H, Yan XX, Xiong K: Beta-amyloid precursor protein cleavage enzyme-1 expression in adult rat retinal neurons in the early period after lead exposure. Neural Regen Res 2011, 6(14):1045-1051.

12. Xiong K, Cai H, Luo XG, Struble RG, Clough RW, Yan XX: Mitochondrial respiratory inhibition and oxidative stress elevate beta-secretase (BACE1) proteins and activity in vivo in the rat retina. Exp Brain Res 2007 181(3):435-446.

13. Misonou $H$, Morishima-Kawashima M, Ihara $Y$ : Oxidative stress induces intracellular accumulation of amyloid beta-protein (Abeta) in human neuroblastoma cells. Biochemistry 2000, 39(23):6951-6959.

14. Paola D, Domenicotti C, Nitti M, Vitali A, Borghi R, Cottalasso D, Zaccheo D, Odetti $P$, Strocchi $P$, Marinari UM, et al: Oxidative stress induces increase in intracellular amyloid beta-protein production and selective activation of betal and betall PKCs in NT2 cells. Biochem Biophys Res Commun 2000, 268(2):642-646.

15. Tamagno E, Bardini P, Obbili A, Vitali A, Borghi R, Zaccheo D, Pronzato MA, Danni $O$, Smith MA, Perry G, et al: Oxidative stress increases expression and activity of BACE in NT2 neurons. Neurobiol Dis 2002, 10(3):279-288.

16. Fukumoto $H$, Cheung $B S$, Hyman BT, Irizarry MC: Beta-secretase protein and activity are increased in the neocortex in Alzheimer disease. Arch Neurol 2002, 59(9):1381-1389.

17. Li R, Lindholm K, Yang LB, Yue X, Citron M, Yan R, Beach T, Sue L, Sabbagh $M$, Cai $H$, et al: Amyloid beta peptide load is correlated with increased beta-secretase activity in sporadic Alzheimer's disease patients. Proc Natl Acad Sci U S A 2004, 101(10):3632-3637.

18. Ji X, Feng YF: Advances in studies on saponins in Anemarrhena asphodeloides. Chinese Traditional and Herbal Drugs 2010, 41(4):S12-S15.

19. Hu Y, Xia Z, Sun Q, Orsi A, Rees D: A new approach to the pharmacological regulation of memory: Sarsasapogenin improves memory by elevating the low muscarinic acetylcholine receptor density in brains of memory-deficit rat models. Brain Res 2005, 1060(1-2):26-39. 
20. Li TJ, Qiu Y, Yang PY, Rui YC, Chen WS: Timosaponin B-II improves memory and learning dysfunction induced by cerebral ischemia in rats. Neurosci Lett 2007, 421(2):147-151.

21. Lu WQ, Qiu Y, Li TJ, Tao X, Sun LN, Chen WS: Timosaponin B-II inhibits pro-inflammatory cytokine induction by lipopolysaccharide in BV2 cells. Arch Pharm Res 2009, 32(9):1301-1308.

22. Ouyang S, Sun LS, Guo SL, Liu X, Xu JP: Effects of timosaponins on learning and memory abilities of rats with dementia induced by lateral cerebral ventricular injection of amyloid beta- peptide. Di Yi Jun Yi Da Xue Xue Bao 2005, 25(2):121-126.

23. Cai F, Sun L, Gao S, Yang Y, Yang Q, Chen W: A rapid and sensitive liquid chromatography-tandem mass spectrometric method for the determination of timosaponin B-II in blood plasma and a study of the pharmacokinetics of saponin in the rat. J Pharm Biomed Anal 2008, 48(5):1411-1416.

24. Lu WQ, Qiu Y, Li TJ, Tao X, Sun LN, Chen WS: Antiplatelet and antithrombotic activities of timosaponin B-II, an extract of Anemarrhena asphodeloides. Clin Exp Pharmacol Physiol 2011, 38(7):430-434.

25. Forstl H, Kurz A: Clinical features of Alzheimer's disease. Eur Arch Psychiatry Clin Neurosci 1999, 249(6):288-290.

26. Backman L, Jones S, Berger AK, Laukka EJ, Small BJ: Multiple cognitive deficits during the transition to Alzheimer's disease. J Intern Med 2004, 256(3):195-204.

27. Frost $\mathrm{S}$, Martins RN, Kanagasingam Y: Ocular biomarkers for early detection of Alzheimer's disease. J Alzheimers Dis 2010, 22(1):1-16.

28. Koronyo-Hamaoui M, Koronyo Y, Ljubimov AV, Miller CA, Ko MK, Black KL, Schwartz M, Farkas DL: Identification of amyloid plaques in retinas from Alzheimer's patients and noninvasive in vivo optical imaging of retinal plaques in a mouse model. Neurolmage 2011, 54(Suppl 1):S204-S217.

29. Chiu K, Chan TF, Wu A, Leung IY, So KF, Chang RC: Neurodegeneration of the retina in mouse models of Alzheimer's disease: what can we learn from the retina? Age (Dordr) 2012, 34(3):633-649.

30. Nunomura A, Perry G, Aliev G, Hirai K, Takeda A, Balraj EK, Jones PK, Ghanbari H, Wataya T, Shimohama S, et al: Oxidative damage is the earliest event in Alzheimer disease. I Neuropathol Exp Neurol 2001, 60(8):759-767.

31. Reed TT, Pierce WM, Markesbery WR, Butterfield DA: Proteomic identification of HNE-bound proteins in early Alzheimer disease: Insights into the role of lipid peroxidation in the progression of AD. Brain Res 2009, 1274:66-76.

32. Smith MA, Zhu X, Tabaton M, Liu G, McKeel DW Jr, Cohen ML, Wang X, Siedlak SL, Dwyer BE, Hayashi T, et al: Increased iron and free radical generation in preclinical Alzheimer disease and mild cognitive impairment. J Alzheimer's Disease: JAD 2010, 19(1):363-372.

33. Lee HP, Pancholi N, Esposito L, Previll LA, Wang X, Zhu X, Smith MA, Lee HG: Early induction of oxidative stress in mouse model of Alzheimer disease with reduced mitochondrial superoxide dismutase activity. PLoS One 2012, 7(1):e28033.

34. Butterfield DA, Sultana R: Methionine-35 of abeta(1-42): importance for oxidative stress in Alzheimer disease. J Amino Acids 2011, 2011:198430.

35. Ma B, Xu Q, Zhao Y, Xiong C, Tan D: Use of timosaponin Bll in the preparation of a medicament or product for the prevention and treatment of stroke. In Google Patents. 2011.

36. Yun D, Bai-ping M, Yu-wen C, Yu-xian S, Jing-jing Z, Yu-jun S: Protective effects of timosaponin Bll on primary neurons against beta amyloid peptide 25-35. Chin Pharmacol Bull 2009, 25(2):244-247.

doi:10.1186/1472-6882-12-189

Cite this article as: Huang et al:: Timosaponin-BII inhibits the upregulation of BACE1 induced by Ferric Chloride in rat retina. BMC Complementary and Alternative Medicine 2012 12:189.

\section{Submit your next manuscript to BioMed Central and take full advantage of:}

- Convenient online submission

- Thorough peer review

- No space constraints or color figure charges

- Immediate publication on acceptance

- Inclusion in PubMed, CAS, Scopus and Google Scholar

- Research which is freely available for redistribution

Submit your manuscript at www.biomedcentral.com/submit 